\title{
Interview
}

\section{Dokumentation erfolgreich entbürokratisiert}

Bei Ein-STEP sind fast 50\% der ambulanten, stationären und teilstationären Pflegeeinrichtungen in der Langezeitpflege registriert. Wir fragten Elisabeth Beikirch, wie sie sich dieses Interesse an der Entbürokratisierung der Pflegedokumentation erklärt.

? Am 31. Oktober 2017 endet das Entbürokratisierungsprojekt in der Pflege "EinSTEP". Warum war es so erfolgreich? Beikirch: Die herkömmliche Dokumentationspraxis empfanden viele Pflegende als extrem belastend. Das erklärt das große Interesse. Es ist auch der Erfolg eines nie dagewesenen intensiven Dialogs zwischen Politik und Pflegenden. Ein weiterer Faktor war das Engagement aller Verbände der Trägerorganisationen. Sie stellten die gesamte Logistik der Implementierungsstrategie auf Bundes- und Landesebene sicher. So konnten über 800 Multiplikatoren geschult werden und den Einrichtungen standen Ressourcen zur Unterstützung zur Verfügung. Eine entscheidende Rolle spielte die klare Botschaft der Medizinischen Dienste, des Prüfdienst der PKV und der Heimaufsichten, die Implementierung bundesweit positiv zu begleiten. Dies hat zu einem verstärkt fachlichen Dialog bei Prüfungen geführt. Entscheidend war auch, dass die Pflegenden die Pflegedokumentation wieder als ein praxistaugliches Instru- ment zur Steuerung des Pflegeprozesses ansahen. Der personzentrierte Ansatz führt zu einer veränderten Gesprächskultur mit der pflegebedürftigen Person. Es geht um die Verständigung zu individuellen Wünschen in der Pflege sowie Aspekten der Selbstbestimmung. Natürlich spielt die Zeitersparnis auch eine Rolle. Das Strukturmodell ist aus dem pflegerischen Alltag nicht mehr wegzudenken. Hierzu trug wesentlich die Klarstellung zu rechtlichen Grundlagen durch die begleitenden juristischen Experten bei.

? Welches waren die größten Stolperfallen bei der Implementierung?

Beikirch: Die rechtzeitige Bereitstellung von Produkten der Hersteller von Dokumentationssystemen, die tatsächlich eine schlanke Pflegedokumentation abbilden. Entscheidend ist, dass die Pflegeeinrichtungen die Einführung sorgfältig planen und inhaltlich wie organisatorisch vorbereiten müssen. Hierfür gibt es einen exemplarischen Projektplan in den Schulungsunterlagen. Die Herausforderung

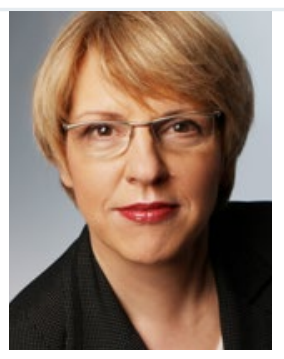

Elisabeth

Beikirch

Fachliche Leitung

Projektbüro

Ein-STEP besteht darin, jahrelange schematische Routinen in der Dokumentationspraxis zu ändern. Größte Stolperfalle ist immer noch der veränderte Umgang mit der Risikoeinschätzung. Wer aus vermeintlichen Sicherheitsgründen an schematische Routinen festhält, beraubt sich eines wesentlichen Effekts des Strukturmodells, nämlich das rechtzeitige Erkennen von Veränderungsprozessen durch ein individuell und anlassbezogenes Vorgehen.

? Wo finden Pflegeeinrichtungen jetzt Unterstützung?

Beikirch: Die Verbände werden ihr Angebot zur Schulung und Begleitung der Pflegeeinrichtungen aufrechterhalten. Zusätzlich zu Inhouseschulungen existieren regionale Netzwerke. Ansprechpartner sind pro Land und Verband auf unserer Homepage zu finden. Diese Kommunikationsplattform wird von den Verbänden ab 1. November 2017 übernommen.

Das Interview führte Heike Ottow

\subsection{Teilnehmer in Berlin erwartet}

— Vom 15.-17. Oktober 2017 findet der World Health Summit in Berlin statt. Der "Gesundheitsgipfel" gilt als das wichtigste strategische Forum für weltweite Gesundheitsfragen. Entsprechend international und hochkarätig ist die Konferenz besetzt: Zu den Sprechern zählen acht Minister, beispielsweise die Gesundheitsminister aus Deutschland, Portugal und Mali, zwei Nobelpreisträger sowie CEOs von Unternehmen wie Bayer, Roche und SAP, von der WHO und von NGOs wie das Internationale Rote Kreuz und Ärzte ohne Grenzen. Die
Veranstaltung steht erneut unter der Schirmherrschaft von Bundeskanzlerin Angela Merkel und dem Präsidenten der Europäischen Kommission Jean-Claude Juncker. Zentrale Themen 2017 sind die Gesundheitspolitik der G7/G20, die Entwicklung neuer Impfstoffe, die Digitalisierung und Big Data, Gesundheitssicherheit, urbane Gesundheit sowie Afrika und die Nachhaltigkeitsziele der Vereinten Nationen.

www.worldhealthsummit.org

\section{Landespflegekammer Neuer Vorstand}

- Der Errichtungsausschusses der Landespflegekammer Niedersachsen besetzte den Vorstand nach: Das Führungsteam besteht aus der Vorsitzenden des Errichtungsausschusses Katrin Havers (Gesundheits- und Kinderkrankenpflegerin), der stellv. Vorsitzenden Sandra Mehmecke (Gesundheitsund Krankenpflegerin) und den Vorstandsmitgliedern Jürgen Butzke (Altenpfleger), Nadya Klarmann (Altenpflegerin) und Rolf Weiß (Gesundheits- und Krankenpfleger).

www.pflegekammer-nds.de 